\title{
On the complexity of computing Kostka numbers and Littlewood-Richardson coefficients
}

\author{
Hariharan Narayanan
}

Received: 2 June 2005 / Accepted: 17 February 2006 / Published online: 11 July 2006

(C) Springer Science + Business Media, LLC 2006

\begin{abstract}
Kostka numbers and Littlewood-Richardson coefficients appear in combinatorics and representation theory. Interest in their computation stems from the fact that they are present in quantum mechanical computations since Wigner [15]. In recent times, there have been a number of algorithms proposed to perform this task $[1-3,11,12]$. The issue of their computational complexity has received attention in the past, and was raised recently by E. Rassart in [11]. We prove that the problem of computing either quantity is $\# P$-complete. Thus, unless $P=N P$, which is widely disbelieved, there do not exist efficient algorithms that compute these numbers.
\end{abstract}

Keywords Kostka numbers · Littlewood-Richardson coefficients · Computational complexity

\section{Introduction}

Let $\mathbb{N}=\{1,2, \ldots\}$ be the set of positive integers and $\mathbb{Z}_{\geq 0}=\mathbb{N} \cup\{0\}$. Let $\lambda=\left(\lambda_{1}, \ldots, \lambda_{s}\right) \in \mathbb{N}^{s}, \lambda_{1} \geq \lambda_{2} \geq \ldots \geq \lambda_{s} \geq 1, \mu=\left(\mu_{1}, \ldots, \mu_{t}\right) \in \mathbb{Z}_{\geq 0}^{t}, v=$ $\left(v_{1}, \cdots, v_{u}\right) \in \mathbb{Z}_{\geq 0}^{u}$ and $\alpha=\left(\alpha_{1}, \ldots, \alpha_{v}\right) \in \mathbb{N}^{v}, \alpha_{1} \geq \cdots \geq \alpha_{v} \geq 1$. The Kostka number $K_{\lambda \mu}$ and the Littlewood-Richardson coefficient $c_{\lambda \alpha}^{\nu}$ play an essential role in the representation theory of the symmetric groups and the special linear groups. Their combinatorial definitions can be found in Section 2. These have been present in quantum mechanical computations since the time of Wigner ([15]). Recently, in [11], E. Rassart asked whether there exist fast (polynomial time) algorithms to compute Kostka numbers and Littlewood Richardson coefficients (Question 1, page 99). We prove that the two quantities are \#P-complete (see Theorems 1,2). It is known that if a

\footnotetext{
H. Narayanan $(\triangle)$

Department of Computer Science,University of Chicago

e-mail: hari@cs.uchicago.edu
} 
$\# P$-complete quantity were computable in polynomial time, $P=N P$. An explanation of this fact is sketched in Section 2. Thus, under the widely believed hypothesis that $P \neq N P$, there do not exist efficient (polynomial time) algorithms to compute Kostka numbers and Littlewood-Richardson coefficients.

In [1], Barvinok and Fomin show how the set of all non-zero $K_{\lambda \mu}$ for a given $\mu$ can be produced in time that is polynomial in the total size of the input and output. They also give a probabilistic algorithm running in time, polynomial in the total size of input and output, that computes the set of all non-zero Littlewood-Richardson coefficients $c_{\lambda \mu}^{\nu}$ given $\lambda$ and $\mu$. In [3], methods for the explicit computation of the Kostka numbers and Littlewood-Richardson coefficients using vector partition functions are discussed. $K_{\lambda \mu}$ is the multiplicity of the weight $\mu$ in the representation $V_{\lambda}$ of the lie algebra $s l_{r+1}(\mathbb{C})$ of the special linear group having highest weight $\lambda$ and $c_{\lambda \alpha}^{\nu}$ is the multiplicity of $V_{v}$ in the tensor product $V_{\lambda} \otimes_{\mathbb{C}} V_{\alpha}$. They also appear in the representation theory of the symmetric groups (see chapter 7, [5]). While there are formulas for $K_{\lambda \mu}$ and $c_{\lambda \alpha}^{\nu}$ due to Kostant and Steinberg respectively ([3], [2]), the number of terms is, in general, exponential in the bit-length of the input. The positivity of $K_{\lambda \mu}$ can be answered in polynomial time (see Proposition 1), and so can the question of whether $c_{\lambda \alpha}^{\nu}>0$, though the latter is a non-trivial fact established by K. Mulmuley and M. Sohoni [8], and uses the proof of the Saturation Conjecture by Knutson and Tao [7]. This fact plays an important role in the approach to the $P$ vs $N P$ question [9] due to K. Mulmuley and M. Sohoni.

We reduce the \#P-complete problem of finding the number $|\mathbb{I}(\mathbf{a}, \mathbf{b})|$ of $2 \times k$ contingency tables to that of finding some Kostka number $K_{\lambda \mu}$. Kostka numbers are known to be also Littlewood-Richardson (LR) coefficients. Thus, their computation reduces to computing some LR coefficient $c_{\lambda \alpha}^{\nu}$, where $\lambda, \mu, \alpha$ and $v$ can be computed in time polynomial in the size of $(\mathbf{a}, \mathbf{b})$. The main tool used in the reduction to finding Kostka numbers is the R-S-K correspondence ([5], pages 40-41) between the set $\mathbb{I}(\mathbf{a}, \mathbf{b})$ of contingency tables and pairs of tableaux having contents $\mathbf{a}$ and $\mathbf{b}$ respectively.

\section{Preliminaries and notation}

$N P$ is the class of decision problems, $e: \cup_{n \in \mathbb{N}}\{0,1\}^{n} \rightarrow\{0,1\}$, for which there exists a polynomial time Turing machine $M$ and a polynomial $p$ such that $(\forall n \in \mathbb{N}),(\forall x \in$ $\left.\{0,1\}^{n}\right), e(x)=1$ if and only if $\exists y, y \in\{0,1\}^{p(n)}$ such that $M$ accepts $\left.(x, y)\right\}$.

The class $\# P$ is the class of functions $f: \cup_{n \in \mathbb{N}}\{0,1\}^{n} \rightarrow \mathbb{Z}_{\geq 0}$, for which there exists a polynomial time Turing machine $M$ and a polynomial $p$ such that $(\forall n \in$ $\mathbb{N}),\left(\forall x \in\{0,1\}^{n}\right), f(x)=\mid\left\{y \in\{0,1\}^{p(n)}\right.$ such that $M$ accepts $\left.(x, y)\right\} \mid$. Valiant defined the counting class \#P in his seminal paper [13]. Many counting problems are naturally in \#P. For example, counting the number of integer points in a polytope, membership queries to which can be answered in polynomial time is a problem in \# $P$.

A problem $W \in N P$ is $N P$-complete, if given a black box that solves instances of $W$ in polynomial time, any problem in $N P$ can be solved in polynomial time. Similarly, a counting problem $X \in \# P$ is \#P-complete if given a black box that provides solutions to instances of $X$ in polynomial time, any problem in the class \#P can be solved in polynomial time. Note that by definition, counting the number of solutions to any problem in $N P$ is in $\# P$. Thus if a $\# P$-complete counting problem could be solved 


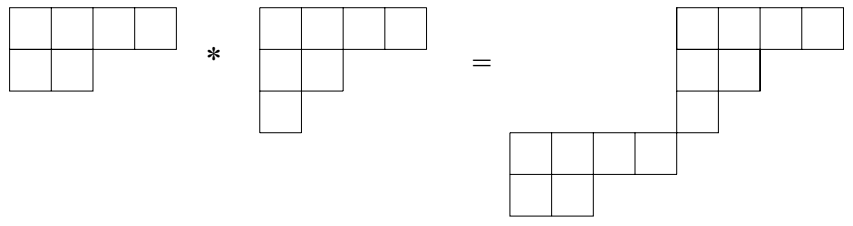

Fig. 1 Left to right, the shapes $\lambda, \alpha$ and the skew shape $\lambda * \alpha$

in polynomial time, we could find the number of solutions to any problem in $N P$ efficiently (in polynomial time.) and thereby solve it, by checking if the number of solutions is $\geq 1$.

The following problem of computing the number of $2 \times k$ contingency tables is known to be $\# P$-complete. Let $\mathbf{a}=\left(a_{1}, a_{2}\right) \in \mathbb{Z}_{\geq 0}, a_{1} \geq a_{2}$ and $\mathbf{b}=\left(b_{1}, \ldots b_{k}\right) \in$ $\mathbb{Z}_{\geq 0}^{k}$. We denote by $\mathbb{I}(a, b)$ the set of $2 \times k$ arrays of nonnegative integers whose row sums are $a_{1}$ and $a_{2}$ respectively and whose column sums are $b_{1}, \ldots, b_{k}$. Geometrically, $\mathbb{I}(a, b)$ can be viewed as the set of integer points in the intersection of the multidimensional rectangular block defined by the column sums, and the diagonal hyperplane given by the first row sum. Counting the number of elements in $\mathbb{I}(a, b)$ was proved to be $\# P$-complete by R. Kannan, M. Dyer and J. Mount in [4].

A Young diagram ([5], page 1) is a collection of boxes, arranged in left justified rows, such that from top to bottom, the number of boxes in a row is monotonically (weakly) decreasing. The first two shapes in Fig. 1 are Young diagrams. A filling is a numbering of the boxes of a Young diagram with positive integers, that are not necessarily distinct. A Young tableau or simply tableau is a filling such that the entries are

1. weakly increasing from left to right across each row, and

2. strictly increasing from top to bottom, down each column.

$P$ and $Q$, in Fig. 2, are Young tableaux. A skew diagram is the diagram obtained from removing a smaller Young diagram out of a larger one. The third shape in Fig. 1 is a skew shape. A skew tableau is a filling of the boxes of a skew diagram with positive integers, non-decreasing in rows, and strictly increasing in columns (see Fig. 5). Let $\lambda:=\left(\lambda_{1}, \ldots, \lambda_{s}\right)$. If the number of boxes in the $i$ th row of a tableau, for $1 \leq i \leq s$ is $\lambda_{i}$, the tableau is said to have shape $\lambda$. If the tableau houses $\mu_{j}$ copies of $j$ for $j \leq t$

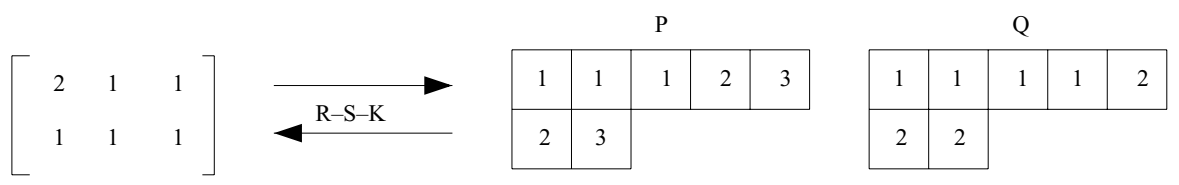

Fig. 2 An instance of the correspondence between $\mathbb{I}(\mathbf{a}, \mathbf{b})$ and $\cup_{\check{\lambda}} \mathbb{T}(\check{\lambda}, \mathbf{a}) \times \mathbb{T}(\check{\lambda}, \mathbf{b})$ for $\mathbf{a}=(4,3), \mathbf{b}=$ $(3,2,2)$ 
and $\mu:=\left(\mu_{1}, \ldots, \mu_{t}\right)$, it is said to have content $\mu$. Thus, in Fig. 2, $P$ and $Q$ have the same shape $(5,2)$, but contents $(3,2,2)$ and $(4,3)$ respectively.

Given two shapes $\lambda$ and $\alpha, \lambda * \alpha$ is defined to be the skew-shape obtained by attaching the lower left corner of $\alpha$ to the upper right corner of $\lambda$ as in Fig. 1 (see [5], page 60). $\operatorname{size}(\lambda, \mu)$ denotes the number of bits used in the description of this tuple of vectors. For $\lambda:=\left(\lambda_{1}, \ldots, \lambda_{s}\right)$, let $|\lambda|=\sum_{i=1}^{s} \lambda_{i}$. For vectors $\lambda, \mu$, we say that $\lambda \unrhd \mu$ if $|\lambda|=|\mu|$ and $\forall i, \sum_{j \leq i} \lambda_{j} \geq \sum_{j \leq i} \mu_{j}$. In addition, if $\lambda \neq \mu$, we say $\lambda \triangleright \mu$. This ordering is called the dominance ordering.

We call a tableau Littlewood-Richardson or $\mathbf{L R}$, if, when its entries are read right to left, top to bottom, at any moment, the number of copies of $i$ that have been encountered is greater than or equal to the number of copies of $i+1$ that have been encountered ([5], page 63). We denote the set of all (possibly skew) tableaux of shape $\lambda$ and content $\mu$ by $\mathbb{T}(\lambda, \mu)$, and its subset consisting of all LR (possibly skew) tableaux by $\operatorname{LRT}(\lambda, \mu)$. The Kostka number $\mathbf{K}_{\lambda \mu}$ is the number of tableaux of shape $\lambda$ and content $\mu$, i.e $|\mathbb{T}(\lambda, \mu)|\left([5]\right.$, page 25). The Littlewood-Richardson coefficient $\mathbf{c}_{\lambda \alpha}^{\nu}$ is the number of LR skew tableaux of shape $\lambda * \alpha$ of content $v$, i.e $|\operatorname{LRT}(\lambda * \alpha, v)|$ (this follows from Corollary 2, (v), page 62 and Lemma 1, page 65 of [5]).

\section{The problems are in \#P}

The particular representation of partitions used above seems to be the most reasonable in the context of computing Kostka numbers and Littlewood-Richardson coefficients. The answer to whether or not a problem is in \#P depends on the format in which the input is specified. If for example, we store partitions by their transposes, then these problems are no longer in the class \# $P$. This can be seen by considering the Kostka number equal to the number of standard tableaux on a $n \times 2$ rectangular array. By the hook length formula, the number of such tableaux is the Catalan number $\left(\begin{array}{c}2 n \\ n\end{array}\right) /(n+1)$ which is exponential in $n$. However if the shape and content were represented as the transposes of the corresponding partitions, they occupy only $O(\log n)$ space. And so the Kostka number is doubly exponential in the size of the input. It is not hard to see that this is impossible for counting problems in the class \#P. On the other hand, if the partitions were represented in unary, it is not clear what the complexity of computing Kostka numbers and LR coefficients is. In unary, the partition $(3,2,1)$ would be represented as $(111,11,1)$. Thus unlike in the binary case, one cannot represent partitions with very large parts efficiently. It is clear that the problems are in $\# P$ for the unary case, but it is not clear whether they are \# $P$-complete.

The tableau shapes $\lambda, \alpha$ and contents $\mu, v$ are described by vectors with integer coefficients. The Littlewood-Richardson coefficient number $c_{\lambda \alpha}^{\nu}$ counts the number of integer points of a polytope of dimension $O\left(\operatorname{size}(\lambda, \mu)^{2}\right)$, given by the intersection of $O\left(\operatorname{size}(\lambda, \mu)^{2}\right)$ halfspaces. The defining coefficients of these halfspaces have size $O(\operatorname{size}(\lambda, \mu))$. This follows from the encoding of relevant skew tableaux in the form of Littlewood-Richardson triangles (see [10].) Therefore the computation of Littlewood-Richardson coefficients is in \#P. The Kostka number $K_{\lambda \mu}$ is known to correspond to Littlewood-Richardson coefficients in parameters whose sizes are polynomial in $\operatorname{size}(\lambda, \mu)$. For the sake of completeness, an explicit correspondence has 
been established in Lemma 2. It follows that the problem of computing the Kostka number $K_{\lambda \mu}$ is in \#P.

Proposition 1. Given $\lambda$ and $\mu$, whether or not $K_{\lambda \mu}>0$ can be answered in polynomial time.

Proof: Let $\lambda, \mu$ be defined as in Section 1. For any permutation $\sigma$ of the set $\{1, \ldots, t\}$, let $\sigma(\mu)$ be the vector $\left(\mu_{\sigma(1)}, \ldots, \mu_{\sigma(t)}\right)$. It is a known fact that $K_{\lambda \mu}=K_{\lambda \sigma(\mu)}$ (see [5], page 26). Let $\sigma$ be a permutation such that $\forall i \leq t-1, \mu_{\sigma(i)} \geq \mu_{\sigma(i+1)}$. For any $\breve{\mu}$, whose components are arranged in non-increasing order, it is known that $K_{\lambda \check{\mu}}>0$ if and only if $\lambda \unrhd \check{\mu}$ (see [5], page 26). Whether $\lambda \unrhd \sigma(\mu)$ can be checked in time that is $O(\operatorname{size}(\lambda, \mu))$. Thus, whether or not $K_{\lambda \mu}>0$ can be answered in time $O(\operatorname{size}(\lambda, \mu) \ln (\operatorname{size}(\lambda, \mu))$, which is the time it takes to find a permutation $\sigma$ that arranges the components of $\mu$ in non-increasing order.

\section{Hardness results}

Lemma 1. Given $\mathbf{a}=\left(a_{1}, a_{2}\right) \in \mathbb{Z}_{\geq 0}^{2}, a_{1} \geq a_{2}$, and $\mathbf{b}=\left(b_{1}, \ldots, b_{k}\right) \in \mathbb{Z}_{\geq 0}^{k}$, let $\lambda=$ $\left(a_{1}+a_{2}, a_{2}\right)$ and $\mu=\left(b_{1}, \ldots, b_{k}, \bar{a}_{2}\right)$. Then, $|\mathbb{I}(\mathbf{a}, \mathbf{b})|=K_{\lambda \mu}$.

Proof: The R-S-K (Robinson-Schensted-Knuth) correspondence ([5], pages 40-41) gives a bijection between $\mathbb{I}(\mathbf{a}, \mathbf{b})$, the set of $2 \times k$ contingency tables with row sums a and column sums $\mathbf{b}$, and pairs of tableaux $\left(T_{1}, T_{2}\right)$ having a common shape but contents $\mathbf{a}$ and $\mathbf{b}$ respectively. In other words, we have a bijection between $\mathbb{I}(\mathbf{a}, \mathbf{b})$ and $\cup_{\check{\lambda}} \mathbb{T}(\check{\lambda}, \mathbf{a}) \times \mathbb{T}(\check{\lambda}, \mathbf{b})$. A sample correspondence is shown in Fig. 2.

Claim 1. For every shape $\check{\lambda}=\left(\check{\lambda}_{1}, \check{\lambda}_{2}\right)$, such that that $\check{\lambda} \unrhd \mathbf{a}$, there is exactly one tableau having shape $\check{\lambda}$ and content a. For any other shape $\check{\lambda}$ there is no tableau having shape $\check{\lambda}$ and content $\mathbf{a}$.

It follows from the proof of Proposition 1 that the existence of a tableau with shape $\check{\lambda}$ and content $\mathbf{a}$ is equivalent to the condition $\check{\lambda} \unrhd \mathbf{a}$. Any tableau with content $\mathbf{a}=$ $\left(a_{1}, a_{2}\right)$ can have at most two rows, since the entries in a single column are all distinct. The filling in which the first $a_{1}$ boxes of the top row contain 1 and all others contain 2 is a tableau (see $Q$ in Fig. 3). Since all the copies of 1 must be in the first row and must be in a contiguous stretch including the leftmost box, this is the only tableau in $\mathbb{T}(\lambda, \mathbf{a})$. Hence the claim is proved.
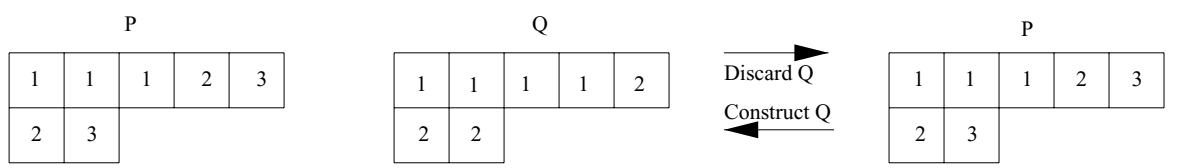

Fig. 3 An instance of the correspondence between $\cup_{\check{\lambda}} \mathbb{T}(\check{\lambda}, \mathbf{a}) \times \mathbb{T}(\check{\lambda}, \mathbf{b})$ and $\cup_{\check{\lambda} \unrhd \mathbf{a}} \mathbb{T}(\check{\lambda}, \mathbf{b})$ for $\mathbf{a}=(4,3)$ and $\mathbf{b}=(3,2,2)$. 


\begin{tabular}{|l|l|l|l|l|}
\hline 1 & 1 & 1 & 2 & 3 \\
\hline 2 & 3 & \multicolumn{3}{|c}{} \\
\hline
\end{tabular}

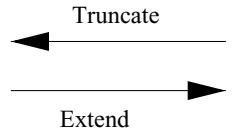

\begin{tabular}{|l|l|l|l|l|l|l|}
\hline 1 & 1 & 1 & 2 & 3 & 4 & 4 \\
\hline 2 & 3 & 4 & \multicolumn{3}{|l}{} \\
\hline
\end{tabular}

Fig. 4 An instance of the correspondence between $\cup_{\check{\lambda} \unrhd \mathbf{a}} \mathbb{T}(\check{\lambda}, \mathbf{b})$ and $\mathbb{T}(\lambda, \mu)$, where $a=(4,3), b=$ $(3,2,2), \lambda=(7,3)$ and $\mu=(3,2,2,3)$

Thus there is a bijection between $\cup_{\check{\lambda}} \mathbb{T}(\check{\lambda}, \mathbf{a}) \times \mathbb{T}(\check{\lambda}, \mathbf{b})$ and the set of tableaux of content $\mathbf{b}$ having some shape $\check{\lambda} \unrhd \mathbf{a}$. i.e, there is a bijection between $\cup_{\check{\lambda}} \mathbb{T}(\check{\lambda}, \mathbf{a}) \times$ $\mathbb{T}(\check{\lambda}, \mathbf{b})$ and $\cup_{\check{\lambda} \unrhd \mathbf{a}} \mathbb{T}(\check{\lambda}, \mathbf{b})$. An example of this is provided in Fig. 3. Let us now consider the set $\cup_{\check{\lambda} \unrhd \mathbf{a}} \mathbb{T}(\check{\bar{\lambda}}, \mathbf{b})$.

Claim 2. Any tableau in $\cup_{\check{\lambda} \unrhd \mathbf{a}} \mathbb{T}(\check{\lambda}, \mathbf{b})$ can be extended to a tableau of the shape $\lambda=$ $\left(a_{1}+a_{2}, a_{2}\right)$ by filling the boxes that are in $\lambda$ but not $\check{\lambda}$, with $k+1$. This extension is a bijection between $\cup_{\check{\lambda} \unrhd \mathbf{a}} \mathbb{T}(\check{\lambda}, \mathbf{b})$ and $\mathbb{T}(\lambda, \mu)$.

If there is a tableau of shape $\check{\lambda}$ and content $\mathbf{a}, \check{\lambda}_{1} \leq a_{1}+a_{2}$, and $\check{\nu_{2}} \leq a_{2} . \check{\lambda} \unrhd \mathbf{a} \Longrightarrow$ $\check{\lambda}_{1} \geq a_{2}=\lambda_{2}$. Therefore no two of the boxes in $\lambda$ which are not in $\check{\lambda}$ belong to the same column. Those of these boxes, that are present in a given row, occupy a contiguous stretch that includes the rightmost box. Therefore by filling them with $k+1$ we get a tableau in $\mathbb{T}(\lambda, \mu)$. Conversely, given a tableau $T$ in $\mathbb{T}(\lambda, \mu)$, deleting all boxes of $T$ filled with $k+1$ gives a tableau in $\cup_{\check{\lambda} \unrhd \mathbf{a}} T(\check{\lambda}, \mathbf{b})$. These two maps are inverses of each other and hence provide a bijection between $\cup_{\check{\lambda} \unrhd \mathbf{a}} T(\check{\lambda}, \mathbf{b})$ and $\mathbb{T}(\lambda, \mu)$. Hence the claim is proved.

An example of this correspondence has been illustrated in Fig. 4. Therefore, $|\mathbb{I}(\mathbf{a}, \mathbf{b})|=\left|\cup_{\check{\lambda}} \mathbb{T}(\check{\lambda}, \mathbf{a}) \times \mathbb{T}(\check{\lambda}, \mathbf{b})\right|=\left|\cup_{\check{\lambda} \unrhd \mathbf{a}} \mathbb{T}(\check{\lambda}, \mathbf{b})\right|=|\mathbb{T}(\lambda, \mu)|=K_{\lambda \mu}$.

Theorem 1. The problem of computing $K_{\lambda \mu}$, even when $\lambda$ has only 2 rows, is \#Pcomplete.

Proof: Computing $K_{\lambda \mu}$ is in \#P as shown in Section 3. Now the result follows from Lemma 1 because the computation of $|\mathbb{I}(\mathbf{a}, \mathbf{b})|$ is known to be $\# P$-complete ([4]).

Lemma 2. Given $\lambda=\left(\lambda_{1}, \lambda_{2}\right) \in \mathbb{Z}_{\geq 0}^{2}, \lambda_{1} \geq \lambda_{2}$, and $\mu=\left(\mu_{1}, \ldots, \mu_{\ell}\right) \in \mathbb{Z}_{\geq 0}^{\ell}$, let $\alpha=\left(\alpha_{1}, \ldots, \alpha_{\ell-1}\right)$ where $(\forall i) \alpha_{i}=\sum_{j>i} \mu_{i}$, and $v=\left(v_{1}, \ldots, v_{\ell}\right)$, where $\forall i \leq \ell-$ $1, v_{i}=\alpha_{i}+\mu_{i}$, and $v_{\ell}=\mu_{\ell}$. Then $K_{\lambda \mu}=c_{\lambda \alpha}^{v}$.

Proof: $c_{\lambda \alpha}^{\nu}$ is, by definition, $|\operatorname{LRT}(\lambda * \alpha, v)|$, which is the number of LR tableaux on the skew shape $\lambda * \alpha$ that have content $\nu$. The skew shape $\lambda * \alpha$ consists of a copy of $\lambda$ and a copy of $\alpha$, as in Figs. 1 and 5. For any skew tableau $S$ of shape $\lambda * \alpha$, we shall denote by $\left.S\right|_{\alpha}$, the restriction of $S$ to the copy of $\alpha$ and by $\left.S\right|_{\lambda}$, the restriction of $S$ to the copy of $\lambda$. Thus, $\left.S\right|_{\alpha}$ is a tableau of shape $\alpha$ and $\left.S\right|_{\lambda}$ is a tableau of shape $\lambda$.

Let $S \in \operatorname{LRT}(\lambda * \alpha, v)$. For $i \leq \ell-1$, it follows from the LR and tableau constraints that the $i$ th row of $\left.S\right|_{\alpha}$ must consist entirely of copies of $i$. 


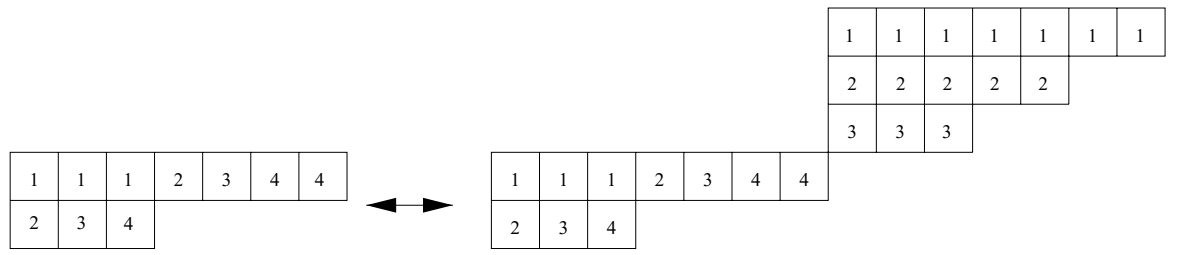

Fig. 5 An instance of the correspondence between $\mathbb{T}(\lambda, \mu)$ and $\operatorname{LR} \mathbb{T}(\lambda * \alpha, v)$ for $\lambda=(7,3)$ and $\mu=$ $(3,2,2,3), \alpha=(7,5,3)$ and $v=(10,7,5,3)$

Consequently, $\left.S\right|_{\lambda}$ must have content $v-\alpha=\mu$. In other words, $\left.S\right|_{\lambda} \in \mathbb{T}(\lambda, \mu)$. Conversely, given any tableau $T \in \mathbb{T}(\lambda, \mu)$, let $S(T)$ be the skew tableau of shape $\lambda * \alpha$ in which $\left.S(T)\right|_{\lambda}=T$ and the $i$ th row of $\left.S(T)\right|_{\alpha}$ consists entirely of copies of $i$. It is not difficult to see that $S(T) \in \operatorname{LR} \mathbb{T}(\lambda * \alpha, v) .\left.S(T)\right|_{\lambda}=T$, thus we have a bijection between $\operatorname{LRT}(\lambda * \alpha, \nu)$, the set of LR skew tableaux of shape $\lambda * \alpha$ having content $\nu$ and $\mathbb{T}(\lambda, \mu)$, the set of tableaux of shape $\lambda$ having content $\mu$. Hence $K_{\lambda \mu}=|\mathbb{T}(\lambda, \mu)|=|\operatorname{LR} \mathbb{T}(\lambda * \alpha, \nu)|=c_{\lambda \alpha}^{\nu}$ as claimed.

Theorem 2. The problem of computing $c_{\lambda \alpha}^{\nu}$, even when $\lambda$ has only 2 rows is \#Pcomplete.

Proof: By the explanation in Section 3, computing $c_{\lambda \alpha}^{v}$ is in $\# P$. We have already proved in Theorem 1, that the computation of $K_{\lambda \mu}$ is $\# P$-complete. The result now follows from Lemma 2.

\section{Conclusion}

We proved that the problems of computing Kostka numbers and LittlewoodRichardson coefficients are $\# P$-complete. The reduction to computing Kostka numbers was from the \#P-complete problem [4] of computing the number of contingency tables having given row and column sums. The problem of computing Kostka numbers was then reduced to that of computing Littlewood-Richardson coefficients. FPRAS (Fully Polynomial Randomized Approximation Schemes) are known to exist for contingency tables with two rows. Thus we obtain FPRAS for a restricted class of Kostka numbers from the correspondence in Lemma 1. It would be of interest to know if such schemes exist for Kostka numbers and Littlewood-Richardson coefficients with general parameters.

Acknowledgements I wish express my gratitude to Ketan Mulmuley for suggesting the topic of this paper and for many valuable discussions. Many thanks are due to Ravi Kannan for informing me about [4]. I also sincerely thank Etienne Rassart, Rahul Santhanam, László Babai and the anonymous referee for helpful comments. 


\section{References}

1. A. Barvinok and S.V. Fomin, Sparse interpolation of symmetric polynomials, Advances in Applied Mathematics 18 (1997), 271-285, MR 98i:05164.

2. S. Billey, V. Guillemin, and E. Rassart, "A vector partition function for the multiplicities of $s l_{k}(\mathbb{C})$," Journal of Algebra 278(1) (2004), 251-293.

3. C. Cochet, Kostka Numbers and Littlewood-Richardson Coefficients, preprint (2003).

4. M. Dyer, R. Kannan, and J. Mount, Sampling contingency tables, Random Structures and Algorithms 10 (1997), 487-506.

5. W. Fulton, "Young Tableaux," London Mathematical Society Student Texts 35 (1997).

6. D. E. Knuth, "Permutations, matrices, and generalized Young tableaux," Pacific Journal of Mathematics 34 (1970), 709-727.

7. A. Knutson and T. Tao, "The honeycomb model of tensor products I: Proof of the saturation conjecture," J. Amer. Math. Soc. 12 (1999), 1055-1090.

8. K. Mulmuley and M, Sohoni, "Geometric Complexity III: on deciding positivity of LittlewoodRichardson coefficients," http://arxiv.org/abs/cs.CC/0501076

9. K. Mulmuley and M. Sohoni, "Geometric complexity theory, P vs. NP, and explicit obstructions.," in Proceedings, International Conference on Algebra and Geometry, Hyderabad (2001).

10. I. Pak and E. Vallejo, "Combinatorics and geometry of Littlewood-Richardson cones," Europ. J. Combinatorics 26 (2005), 995-1008.

11. E. Rassart, "Geometric approaches to computing Kostka numbers and Littlewood-Richardson coefficients," Thesis for the Ph.D. degree in Mathematics, Massachusetts Institute of Technology (MIT), 2004.

12. E. Rassart, “A polynomiality property for Littlewood-Richardson coefficients," Journal of Combinatorial Theory, Series A 107(2) (2004), 161-179.

13. L.G. Valiant, "The complexity of computing the permanent," Theoret. Comp. Sci. 8 (1979), 189-201.

14. M.A.A. van Leeuwen, "The Littlewood-Richardson rule, and related combinatorics," Math. Soc. of Japan Memoirs 11, Interaction of Combinatorics and Representation Theory; arXiv:math.CO/9908099.

15. E. Wigner, "On the consequences of the symmetry of the nuclear Hamiltonian on the spectroscopy of the nuclei," Physical Review 51 (1937), 106-119. 